\title{
The Gravitational Two-Loop Counterterm is Asymptotically Safe
}

\author{
Holger Gies, ${ }^{1, *}$ Benjamin Knorr, ${ }^{1, \dagger}{ }^{\dagger}$ Stefan Lippoldt,,${ }^{1,}$ and Frank Saueressig ${ }^{2}, \S$ \\ ${ }^{1}$ Theoretisch-Physikalisches Institut, Abbe Center of Photonics, \\ Friedrich-Schiller-Universität Jena, Max-Wien-Platz 1, D-07743 Jena, Germany \\ ${ }^{2}$ Institute for Mathematics, Astrophysics and Particle Physics (IMAPP), \\ Radboud University Nijmegen, Heyendaalseweg 135, 6525 AJ Nijmegen, The Netherlands
}

\begin{abstract}
Weinberg's asymptotic safety scenario provides an elegant mechanism to construct a quantum theory of gravity within the framework of quantum field theory based on a non-Gaußian fixed point of the renormalization group flow. In this work we report novel evidence for the validity of this scenario, using functional renormalization group techniques to determine the renormalization group flow of the Einstein-Hilbert action supplemented by the two-loop counterterm found by Goroff and Sagnotti. The resulting system of beta functions comprises three scale-dependent coupling constants and exhibits a non-Gaußian fixed point which constitutes the natural extension of the one found at the level of the Einstein-Hilbert action. The fixed point exhibits two ultraviolet attractive and one repulsive direction supporting a low-dimensional UV-critical hypersurface. Our result vanquishes the longstanding criticism that asymptotic safety will not survive once a "proper perturbative counterterm" is included in the projection space.
\end{abstract}

\section{INTRODUCTION}

General relativity, based on the Einstein-Hilbert action, provides a highly successful classical description of gravitational phenomena from sub-millimeter to cosmic scales. A central puzzle for the construction of a consistent quantum theory of gravity is its perturbative nonrenormalizability. This is manifested by the fact, that an expansion in terms of Newton's constant about flat spacetime gives rise to a divergence at two-loop order. This spoils meaningful predictions for $S$-matrix elements, unless a Goroff-Sagnotti counter-term of the form [1-3]

$$
\Gamma_{\mathrm{GS}}=\frac{1}{\epsilon} \frac{209}{2880} \frac{1}{\left(16 \pi^{2}\right)^{2}} \int d^{4} x \sqrt{g} C_{\mu \nu}{ }^{\kappa \lambda} C_{\kappa \lambda}{ }^{\rho \sigma} C_{\rho \sigma}{ }^{\mu \nu}
$$

with the Weyl-tensor $C_{\mu \nu \rho \sigma}$, is added to the bare action in order to cancel the divergence (in dimensional regularization). In combination with power-counting arguments, this is taken as a signal that an infinite number of counterterms is needed for rendering the full perturbative expansion meaningful. Since renormalization theory relates each counterterm to a free parameter to be fixed from experimental data, the appearance of the GoroffSagnotti term suggests that the perturbative quantization of the Einstein-Hilbert action requires fixing infinitely many parameters. This observation is often interpreted as evidence that conventional quantization of gravity is doomed to fail.

The presence of the counterterm (1) triggered the investigation of a variety of alternative routes towards quantizing gravity, e.g., by modifying the quantization rules, changing the fundamental degrees of freedom, or abandoning local quantum field theory altogether as a fundamental framework for quantum gravity [4-6]. Ultimately, any consistent quantum gravity theory allowing for a classical limit containing Einstein's theory of gravity, as well as its semi-classical extension as a low-energy effective theory for quantized gravitons, has to clarify the fate of the divergencies related to the Goroff-Sagnotti term.

This requirement is more than a technical necessity, as renormalizability - beyond being a strategy of handling divergencies - is a statement about the separability of low-energy observables from physics at highest energy scales. For instance, quantum gravity scenarios that start from discretized building blocks of spacetime and a fundamental length scale may render all divergencies finite. Still, $S$-matrix elements would generically receive large contributions from potentially large higher-order operators, requiring to fix a substantial, if not infinite, set of physical parameters.

At first sight, a natural solution appears to be that the divergencies cancel, e.g., because of a new symmetry at a more fundamental level. While certainly possible, the problem of separation of low-energy physics from highest energy scales may come in again through the backdoor, as this symmetry has to be broken (or restored) at low energy potentially requiring a fine-tuned separation of scales and a large number of parameters.

An indiscriminate association of (1) with quantum field theory approaches to quantum gravity ignores the fact that the Wilsonian viewpoint of renormalization already offers a solution to this puzzle: higher-dimensional operators decouple from the low-energy physics proportional to an inverse power of a high scale $\Lambda$, provided such operators do not acquire large anomalous dimensions. For instance, the $C^{3}$ operator in (1) would be expected to decouple $\sim 1 / \Lambda^{2}$ if the anomalous dimension was small. If so, the $1 / \epsilon$-pole may merely indicate a subleading log-correction as sensed by dimensional regularization. This may sound like a circular argument, as such conclusions can only be drawn in perturbation theory after the theory has been renormalized. Nevertheless, the Wilsonian viewpoint is known to hold also in systems 
with a similar breakdown of perturbative quantization, where a well-controllable ultraviolet limit is facilitated by the existence of an interacting renormalization group (RG) fixed point [7-9].

In the present letter, we provide novel evidence that the Goroff-Sagnotti term is indeed an irrelevant operator from the Wilsonian viewpoint. Our results demonstrate that the challenge posed by the perturbative twoloop analysis is solved by a renormalization flow that decouples the high-scale physics from (semi-)classical Einsteinian low-energy gravity in much the same way as in conventional quantum field theories. For this, we determine the decoupling of the Goroff-Sagnotti term towards the IR quantitatively.

The new ingredient compared to the perturbative analysis is the investigation of the RG flow beyond the perturbative Gaußian fixed point (GFP). In fact, our results confirm the existence of an interacting non-Gaußian fixed point (NGFP) that controls the high-energy limit of gravity, as required for the asymptotic safety scenario [8-14]. By now, the existence of a suitable NGFP has been established within many approximations [15-35]. In particular, it has been shown in the case of gravity coupled to scalar matter that the asymptotic safety mechanism remains intact once the one-loop counterterm is included $[22,23]$. Paralleling this observation, we establish that the Goroff-Sagnotti term supplements only a subdominant quantitative correction to the high-energy behavior of pure gravity: the $C^{3}$ operator approaches an interacting fixed point in the UV and becomes irrelevant towards the IR at an even enhanced rate compared to canonical scaling.

This demonstrates that the asymptotic safety scenario for quantum gravity can solve this long-standing puzzle in a constructive and quantifiable manner, disclosing the two-loop divergence of (1) as a mere perturbative artifact.

\section{FUNCTIONAL RENORMALIZATION}

A powerful tool to investigate renormalizability based on interacting RG fixed points is provided by the functional RG. In the incarnation based on the effective average action $\Gamma_{k}$ [36], the functional RG equation

$$
k \partial_{k} \Gamma_{k}=\frac{1}{2} \operatorname{Str}\left[\left(\Gamma_{k}^{(2)}+\mathcal{R}_{k}\right)^{-1} k \partial_{k} \mathcal{R}_{k}\right]
$$

realizes Wilson's idea of renormalization by integrating out quantum fluctuations shell-by-shell in momentum space. The use of the two-point correlator $\Gamma_{k}^{(2)}$ leads to a formally exact equation. Owed to the regulator $\mathcal{R}_{k}$ the change of $\Gamma_{k}$ is driven by quantum fluctuations with momenta close to $k$. A key advantage of the functional RG is that it permits approximations which do not rely on a small expansion parameter. Moreover, the flow equation allows investigating RG flows without the need of specifying a fundamental action a priori. This makes the setup predestined for searching for fixed points of the renormalization flow beyond the realm of perturbation theory.

We focus on the case where the gravitational degrees of freedom are carried by the spacetime metric $g_{\mu \nu}$. The flow equation (2) can then be constructed via the background-field method splitting the full metric in a background metric $\bar{g}_{\mu \nu}$ and fluctuations $h_{\mu \nu}$, $g_{\mu \nu}=\bar{g}_{\mu \nu}+h_{\mu \nu}$ [10]. In this way the effective average action can be computed in a covariant way.

\section{PROJECTION OF THE FLOW EQUATION}

We study the gravitational renormalization flow projected on the Einstein-Hilbert action supplemented by the two-loop counterterm (1). Our ansatz for the gravitational part of the effective average action, closely following [3], reads

$$
\Gamma_{k}=\Gamma_{k}^{\mathrm{EH}}+\Gamma_{k}^{\mathrm{GS}} .
$$

Here

$$
\Gamma_{k}^{\mathrm{EH}}=\frac{1}{16 \pi G_{k}} \int d^{4} x \sqrt{g}\left(-R+2 \Lambda_{k}\right)
$$

is the Einstein-Hilbert action including a scale-dependent Newton's constant and cosmological constant, and

$$
\Gamma_{k}^{\mathrm{GS}}=\bar{\sigma}_{k} \int d^{4} x \sqrt{g} C_{\alpha \beta}{ }^{\mu \nu} C_{\mu \nu}{ }^{\rho \sigma} C_{\rho \sigma}{ }^{\alpha \beta}
$$

is the two-loop counterterm found by Goroff and Sagnotti with a scale-dependent coupling $\bar{\sigma}_{k}$. The gravitational part of the effective average action is supplemented by a standard gauge-fixing procedure and we adhere to the harmonic gauge used in [10]. The perturbative result (1) suggests that $\bar{\sigma}_{k}$ diverges at least as $\ln k$ for $k \rightarrow \infty$ even in the flat-space on-shell limit $\Lambda_{k} \rightarrow 0$ and after the Newton coupling has been renormalized.

The RG flow of the couplings is found by substituting the ansatz (3) into Eq. (2) and computing the coefficients multiplying the curvature terms appearing in Eqs. (4) and (5). The evaluation of the trace utilizes the technology of the universal RG machine [37] together with off-diagonal heat-kernel methods [38-42].

Two crucial features make this formidable computation feasible: firstly, we use the Ricci scalar, Ricci tensor, and Weyl tensor to construct a basis for the interaction monomials containing a fixed number of covariant derivatives. The second functional derivative of (5) results in a sum of terms containing at least one power of the Weyl tensor. Since $C$ is trace-free by construction, all its contractions with the metric vanish. This entails that 
there is no feedback of the Goroff-Sagnotti term on the renormalization flow of Newton's constant and the cosmological constant. We conclude already at this point that the asymptotic safety properties observed in the Einstein-Hilbert sector are stable upon the inclusion of the Goroff-Sagnotti term. Secondly, the contribution of the Goroff-Sagnotti term to the two-point correlator is of the form $\bar{\sigma}_{k}(C+$ higher powers of the curvature). This structure implies that the $\beta$ function encoding the flow of $\bar{\sigma}_{k}$ is a cubic in $\bar{\sigma}_{k}$ with coefficients depending on Newton's constant and the cosmological constant. As a cubic has at least one zero, also the Goroff-Sagnotti coupling must have a fixed point and hence the associated dimensionless coupling does not necessarily diverge for $k \rightarrow \infty$. The remaining crucial question is whether the $C^{3}$ term is a relevant (as suggested by perturbation theory) or an irrelevant operator. In case of irrelevance, the GoroffSagnotti term does neither require the fixing of an additional physical parameter nor induces a proliferation of counterterms.

In order to determine the coefficients of this cubic it suffices to isolate the term $\sim C^{3}$ from the trace of Eq. (2). As the curvature terms are orthogonal, any term containing a Ricci scalar or Ricci tensor will not contribute to $C^{3}$ and it is sufficient to keep track of powers of the Weyl tensor and its covariant derivatives. Formally, this can be achieved with a background metric $\bar{g}_{\mu \nu}$ of a Ricci-flat $K 3$-surface. Terms contributing to the basis monomial Eq. (5) appear in three different tensor structures which are related by

$$
\begin{aligned}
C^{\alpha}{ }_{\mu}{ }^{\beta}{ }_{\nu} C^{\mu}{ }_{\rho}{ }^{\nu}{ }_{\sigma} C^{\rho}{ }_{\alpha}{ }^{\sigma}{ }_{\beta} & =\frac{1}{2} C_{\mu \nu}{ }^{\rho \sigma} C_{\rho \sigma}{ }^{\alpha \beta} C_{\alpha \beta}{ }^{\mu \nu}, \\
C_{\mu \nu \rho \sigma} D^{2} C^{\mu \nu \rho \sigma} & \simeq-3 C_{\mu \nu}{ }^{\rho \sigma} C_{\rho \sigma}{ }^{\alpha \beta} C_{\alpha \beta}{ }^{\mu \nu},
\end{aligned}
$$

where $\simeq$ denotes that the identity holds up to terms containing the Ricci scalar and Ricci tensor. The vertices entering the computation have been constructed with the Mathematica package xAct [43-48]. Employing the simplifications of a $K 3$-background, the Goroff-Sagnotti vertex contains 900 terms whereas the Einstein-Hilbert vertex has only one term. The computation was done with xAct within one month of CPU time on a core with 2.8 GHz. Most of the CPU time is used for the two vertex diagram due to the enormous number of terms generated by the product rule for covariant derivatives. This makes the present computation quite formidable and complementary to the recent progress in vertex expansions of quantum gravity on flat spacetime [29] of similar complexity.

\section{$\beta$ FUNCTIONS}

The RG flow resulting from the ansatz (3) is conveniently written in terms of dimensionless couplings

$$
\begin{aligned}
g_{i} & \equiv\{\lambda, g, \sigma\}, \\
\lambda & \equiv \Lambda_{k} k^{-2}, \quad g \equiv G_{k} k^{2}, \quad \sigma \equiv \bar{\sigma}_{k} k^{2},
\end{aligned}
$$

and expressed in terms of the $\beta$ functions

$$
k \partial_{k} g_{i} \equiv \beta_{g_{i}}(\lambda, g, \sigma) .
$$

The $\beta$ functions for the dimensionless Newton's constant and cosmological constant have been known since the beginning of the asymptotic safety program [10]. In four spacetime dimensions and for the Litim regulator [49] they read

$$
\begin{aligned}
& \beta_{g}=\left(2+\eta_{N}\right) g, \\
& \beta_{\lambda}=\left(\eta_{N}-2\right) \lambda+\frac{g}{2 \pi}\left(\frac{5}{1-2 \lambda}-4-\frac{5}{6} \eta_{N} \frac{1}{1-2 \lambda}\right) .
\end{aligned}
$$

Here $\eta_{N}$ denotes the anomalous dimension of Newton's constant,

$$
\eta_{N}=\frac{g B_{1}}{1-g B_{2}}
$$

with

$$
\begin{aligned}
& B_{1}=\frac{1}{3 \pi}\left(\frac{5}{1-2 \lambda}-\frac{9}{(1-2 \lambda)^{2}}-5\right) \\
& B_{2}=-\frac{1}{6 \pi}\left(\frac{5}{2} \frac{1}{(1-2 \lambda)}-\frac{3}{(1-2 \lambda)^{2}}\right)
\end{aligned}
$$

The ansatz (3) complements this system by a $\beta$ function for $\sigma$,

$$
\beta_{\sigma}=c_{0}+\left(2+c_{1}\right) \sigma+c_{2} \sigma^{2}+c_{3} \sigma^{3},
$$

where the coefficients $c_{i}(g, \lambda)$ are given by

$$
\begin{aligned}
& c_{0}=\frac{1}{64 \pi^{2}(1-2 \lambda)}\left(\frac{2-\eta_{N}}{2(1-2 \lambda)}+\frac{6-\eta_{N}}{(1-2 \lambda)^{3}}-\frac{5 \eta_{N}}{378}\right), \\
& c_{1}=\frac{3 g}{16 \pi(1-2 \lambda)^{2}}\left(5\left(6-\eta_{N}\right)+\frac{23\left(8-\eta_{N}\right)}{8(1-2 \lambda)}-\frac{7\left(10-\eta_{N}\right)}{10(1-2 \lambda)^{2}}\right) \\
& c_{2}=\frac{g^{2}}{2(1-2 \lambda)^{3}}\left(\frac{233\left(12-\eta_{N}\right)}{10}-\frac{9\left(14-\eta_{N}\right)}{7(1-2 \lambda)}\right) \\
& c_{3}=\frac{6 \pi g^{3}\left(18-\eta_{N}\right)}{(1-2 \lambda)^{4}} .
\end{aligned}
$$

We emphasize that the highest-order coefficient $c_{3}$ is positive for any admissible $\lambda$, positive Newton coupling $g>0$, and $\eta_{N}<18$. We have verified that $c_{3}$ is gauge independent, and that its positivity is independent of the metric parametrization [50-56]. The $\beta$ function Eq. (12) is computed for the first time and constitutes the main result of this work.

\section{FIXED POINTS AND RG FLOW}

The Wilsonian viewpoint links renormalizability to fixed points $g_{i, *}$ of the underlying RG flow where $\left.\beta_{g_{i}}\right|_{g_{j, *}}=0$. Linearizing the $\beta$ functions at a fixed point, local properties of the flow are encoded in the stability 
coefficients $\theta$ defined as minus the eigenvalues of the stability matrix $\left.B_{i j} \equiv \partial_{g_{j}} \beta_{g_{i}}\right|_{g_{*}}$. Relevant directions, corresponding to free parameters of the theory to be fixed by experiment, are associated with stability coefficients with a positive real part.

Already the first calculations $[10,57,58]$ revealed that the system (9) exhibits a GFP and an NGFP

$$
\begin{array}{lll}
\mathrm{GFP}^{\mathrm{EH}}: & \lambda_{*}=0, & g_{*}=0 \\
\mathrm{NGFP}^{\mathrm{EH}}: & \lambda_{*}=0.193, & g_{*}=0.707 .
\end{array}
$$

The GFP corresponds to a free theory and is a saddlepoint: trajectories with a positive Newton coupling do not end at the GFP at high energies, reflecting the perturbative non-renormalizability of the Einstein-Hilbert action in the Wilsonian framework. The NGFP exhibits a complex pair of stability coefficients

$$
\theta_{1,2}=1.475 \pm 3.043 i \text {. }
$$

Thus the NGFP is UV attractive for both Newton's constant and the cosmological constant making it suitable for asymptotic safety.

The $\beta$ function (12) clarifies the fate of the fixed point structure (14) once the counterterm (5) is taken into account. Substituting $\lambda_{*}=g_{*}=0$ into the $\beta$ function for $\sigma$ shows that the $\mathrm{GFP}^{\mathrm{EH}}$ is mapped to

$$
\mathrm{GFP}^{\mathrm{GS}}: \quad \lambda_{*}=0, g_{*}=0, \sigma_{*}=-\frac{7}{128 \pi^{2}} .
$$

The stability coefficients of this fixed point coincide with the classical mass dimension of the coupling constants; the GFP remains a saddle point.

Focusing on the NGFP, it is illuminating to first study the Einstein-Hilbert induced approximation of the $\beta$ function, where only the terms originating from (4) contribute to the running of $\sigma$. Since the contribution of the counterterm to the $\beta$ function (12) is captured by the coefficients $c_{1}, c_{2}$, and $c_{3}$ this approximation corresponds to setting $c_{1}=c_{2}=c_{3}=0$. In this limit the flow has a unique fixed point solution

$$
\operatorname{sGFP}^{\mathrm{GS}}: \lambda_{*}=0.193, g_{*}=0.707, \sigma_{*}=-0.049,
$$

with stability coefficient $\theta_{3}=-2$. This is the analogue of the Gaußian fixed point for $\sigma$ shifted by the finite interactions of $g$ and $\lambda$ at the NGFP (14). The stability coefficient indicates that the new direction is irrelevant in agreement with power-counting arguments.

Taking into account the full non-linear contributions from the $C^{3}$ term, the cubic (12) again has exactly one real root

$$
\mathrm{NGFP}^{\mathrm{GS}}: \lambda_{*}=0.193, g_{*}=0.707, \sigma_{*}=-0.305,
$$

extending the NGFP known from the Einstein-Hilbert projection. The new stability coefficient $\theta_{3}=-79.39$ is again negative, so that the new direction exhibits an
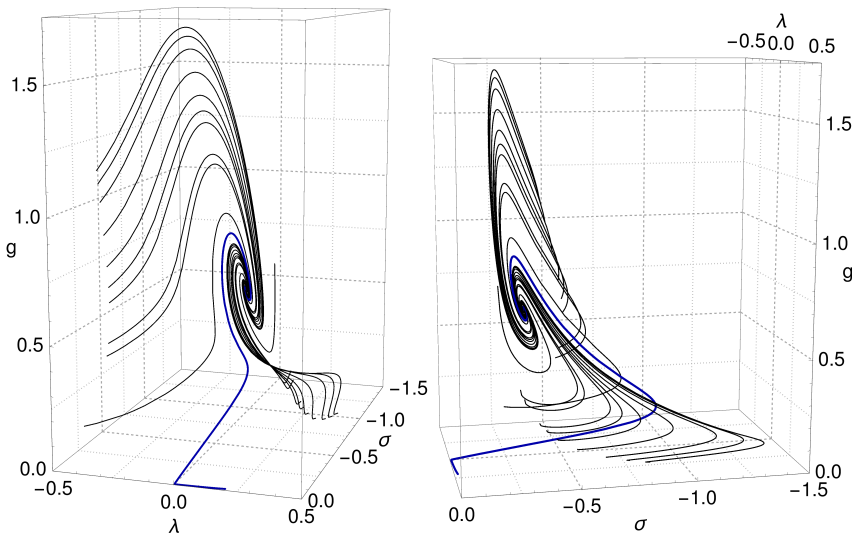

Figure 1. Phase diagram in $(g, \lambda, \sigma)$ space from two perspectives depicting trajectories emanating from the NGFP. The thick line marks a trajectory with a long semi-classical regime near the GFP.

even enhanced irrelevance. In fact, the positivity of $c_{3}$ ensures that $\sigma$ always has a fixed point for which $C^{3}$ is an irrelevant perturbation.

Fig. 1 shows the phase diagram in the theory space spanned by $(g, \lambda, \sigma)$. The flow is governed by the interplay of the GFP (16) and the NGFP (18). The left panel depicts a $(g, \lambda)$ perspective illustrating that the inclusion of the Goroff-Sagnotti term leaves asymptotic safety as observed with the Einstein-Hilbert ansatz [10, 58] and the $R^{2}$-extension [24] fully intact. The thick line exemplifies a trajectory which crosses over from the NGFP at high energies to the GFP at low energies. In the vicinity of the GFP the trajectory develops a long semi-classical regime where the couplings scale classically. The right panel presents a $(g, \sigma)$ perspective; following the semiclassical trajectory towards higher energies, we observe that the Goroff-Sagnotti coupling is first enhanced but then also attracted by NGFP in the deep UV. The dimensionful Goroff-Sagnotti coupling $\bar{\sigma}_{k} \rightarrow \sigma_{*} / k^{2}$ hence vanishes asymptotically for $k \rightarrow \infty$.

While the present ansatz (3) and calculation scheme give a unique answer (18) for the fixed point, the number of real roots of the cubic $\beta$ function (12) depends sensitively on the fixed point values for $g$ and $\lambda$. The inclusion of higher-order operators thus has the potential to yield three fixed points. This does, however, not change our conclusion about the irrelevance of the GoroffSagnotti term, as two of these fixed points have properties equivalent to those discussed above. As an example, let us consider the case where we neglect the cosmological constant, setting $\lambda=0$ at all scales. Then, the NGFP for Newton's constant has three extensions to the $g, \sigma$-plane. The one corresponding to (18) is located at $g_{*}=12 \pi / 23 \simeq 1.639, \sigma_{*}=-0.226$ and has stability coefficients $\theta_{1}=23 / 11 \simeq 2.09$ and $\theta_{3}=-77.38$. 
A second fixed point with the same $g_{*}$ and $\theta_{1}$ corresponds to the shifted Gaußian fixed point for the GoroffSagnotti coupling with $\sigma_{*}=-0.0023$ and stability coefficient $\theta_{3}=-6.06$. This confirms the existence of the NGFP also in the zero-cosmological constant case analyzed by Goroff and Sagnotti.

\section{CONCLUSIONS}

We have studied the non-perturbative renormalization flow of gravity projected onto the Einstein-Hilbert action supplemented by the two-loop counterterm found by Goroff and Sagnotti. All versions of the $\beta$ functions including the Einstein-Hilbert induced approximation, the zero cosmological-constant limit, and the $\beta$ functions including the full feedback of the counterterm, possess a nonGaußian fixed point in agreement with the gravitational asymptotic safety scenario. This settles a long-standing question demonstrating that this perturbative counterterm does not have the power to destroy the non-Gaußian fixed point seen in the Einstein-Hilbert projection. Also the existence of trajectories with a (semi-)classical lowenergy regime is left untouched. Together with the recent construction of fixed functionals [25, 59-71], the verification of locality [29], and first steps towards clarifying unitarity [72], this constitutes hard evidence that the asymptotic safety program indeed can give rise to a consistent quantum theory of gravity within the framework of quantum field theory along the lines envisioned by Weinberg [9].

We thank M. Ammon, K. Krasnov, A. Wipf, and O. Zanusso for interesting discussions. S. L. and F. S. thank the organizers of the "Quantum Gravity in Paris 2015 " workshop for hospitality during the initial stage of the project. The research of F. S. is supported by the Netherlands Organisation for Scientific Research (NWO) within the Foundation for Fundamental Research on Matter (FOM) grants 13PR3137 and 13VP12. H. G., B. K. and S. L. acknowledge support by the DFG under grants No. GRK1523/2, Gi328/7-1, and Wi 777/11-1.

* holger.gies@uni-jena.de

† benjamin.knorr@uni-jena.de

‡ stefan.lippoldt@uni-jena.de

$\S$ f.saueressig@science.ru.nl

[1] Marc H. Goroff and Augusto Sagnotti, "QUANTUM GRAVITY AT TWO LOOPS," Phys. Lett. B160, 81 (1985).

[2] Marc H. Goroff and Augusto Sagnotti, "The Ultraviolet Behavior of Einstein Gravity," Nucl. Phys. B266, 709 (1986).

[3] A. E. M. van de Ven, "Two loop quantum gravity," Conference on Strings and Symmetries Stony Brook, New
York, May 20-25, 1991, Nucl. Phys. B378, 309-366 (1992).

[4] Abhay Ashtekar, "The Winding road to quantum gravity," Curr. Sci. 88, 2064-2074 (2005).

[5] Herbert W. Hamber, Quantum gravitation: The Feynman path integral approach (2009).

[6] Claus Kiefer, Quantum gravity, International series of monographs on physics, Vol. 155 (Oxford Univ. Pr., Oxford, UK, 2012).

[7] Kenneth G. Wilson, "Quantum field theory models in less than four-dimensions," Phys. Rev. D7, 2911-2926 (1973).

[8] Steven Weinberg, "Critical Phenomena for Field Theorists," in Erice Subnucl.Phys.1976:1 (1976) p. 1.

[9] Steven Weinberg, "ULTRAVIOLET DIVERGENCES IN QUANTUM THEORIES OF GRAVITATION," General Relativity: An Einstein centenary survey, Eds. Hawking, S.W., Israel, W; Cambridge University Press , 790-831 (1979).

[10] M. Reuter, "Nonperturbative evolution equation for quantum gravity," Phys.Rev. D57, 971-985 (1998), arXiv:hep-th/9605030 [hep-th].

[11] Max Niedermaier and Martin Reuter, "The Asymptotic Safety Scenario in Quantum Gravity," Living Rev.Rel. 9, 5-173 (2006).

[12] Alessandro Codello, Roberto Percacci, and Christoph Rahmede, "Investigating the Ultraviolet Properties of Gravity with a Wilsonian Renormalization Group Equation," Annals Phys. 324, 414-469 (2009), arXiv:0805.2909 [hep-th].

[13] Daniel F. Litim, "Renormalisation group and the Planck scale," Phil. Trans. Roy. Soc. Lond. A369, 2759-2778 (2011), arXiv:1102.4624 [hep-th].

[14] Martin Reuter and Frank Saueressig, "Quantum Einstein Gravity," New J.Phys. 14, 055022 (2012), arXiv:1202.2274 [hep-th].

[15] O. Lauscher and M. Reuter, "Is quantum Einstein gravity nonperturbatively renormalizable?" Class. Quant. Grav. 19, 483-492 (2002), arXiv:hep-th/0110021 [hep-th].

[16] O. Lauscher and M. Reuter, "Flow equation of quantum Einstein gravity in a higher derivative truncation," Phys. Rev. D66, 025026 (2002), arXiv:hep-th/0205062 [hep-th].

[17] Alessandro Codello and Roberto Percacci, "Fixed points of higher derivative gravity," Phys. Rev. Lett. 97, 221301 (2006), arXiv:hep-th/0607128 [hep-th].

[18] Alessandro Codello, Roberto Percacci, and Christoph Rahmede, "Ultraviolet properties of $\mathrm{f}(\mathrm{R})$-gravity," Int.J.Mod.Phys. A23, 143-150 (2008), arXiv:0705.1769 [hep-th].

[19] Pedro F. Machado and Frank Saueressig, "On the renormalization group flow of $\mathrm{f}(\mathrm{R})$-gravity," Phys.Rev. D77, 124045 (2008), arXiv:0712.0445 [hep-th].

[20] Alfio Bonanno, Adriano Contillo, and Roberto Percacci, "Inflationary solutions in asymptotically safe f(R) theories," Class. Quant. Grav. 28, 145026 (2011), arXiv:1006.0192 [gr-qc].

[21] K. Falls, D.F. Litim, K. Nikolakopoulos, and C. Rahmede, "A bootstrap towards asymptotic safety," (2013), arXiv:1301.4191 [hep-th].

[22] Dario Benedetti, Pedro F. Machado, and Frank Saueressig, "Asymptotic safety in higher-derivative gravity," Mod. Phys. Lett. A24, 2233-2241 (2009), arXiv:0901.2984 [hep-th].

[23] Dario Benedetti, Pedro F. Machado, and Frank Sauer- 
essig, "Taming perturbative divergences in asymptotically safe gravity," Nucl. Phys. B824, 168-191 (2010), arXiv:0902.4630 [hep-th].

[24] Stefan Rechenberger and Frank Saueressig, "The $R^{2}$ phase-diagram of QEG and its spectral dimension," Phys. Rev. D86, 024018 (2012), arXiv:1206.0657 [hep-th].

[25] Maximilian Demmel, Frank Saueressig, and Omar Zanusso, "A proper fixed functional for four-dimensional Quantum Einstein Gravity," JHEP 08, 113 (2015), arXiv:1504.07656 [hep-th].

[26] Astrid Eichhorn, Holger Gies, and Michael M. Scherer, "Asymptotically free scalar curvature-ghost coupling in Quantum Einstein Gravity," Phys. Rev. D80, 104003 (2009), arXiv:0907.1828 [hep-th].

[27] Kai Groh and Frank Saueressig, "Ghost wave-function renormalization in Asymptotically Safe Quantum Gravity," J. Phys. A43, 365403 (2010), arXiv:1001.5032 [hepth].

[28] Astrid Eichhorn and Holger Gies, "Ghost anomalous dimension in asymptotically safe quantum gravity," Phys. Rev. D81, 104010 (2010), arXiv:1001.5033 [hep-th].

[29] Nicolai Christiansen, Benjamin Knorr, Jan Meibohm, Jan M. Pawlowski, and Manuel Reichert, "Local Quantum Gravity," Phys. Rev. D92, 121501 (2015), arXiv:1506.07016 [hep-th].

[30] Andreas Nink and Martin Reuter, "On the physical mechanism underlying Asymptotic Safety," JHEP 01, 062 (2013), arXiv:1208.0031 [hep-th].

[31] Nicolai Christiansen, Daniel F. Litim, Jan M. Pawlowski, and Andreas Rodigast, "Fixed points and infrared completion of quantum gravity," Phys.Lett. B728, 114-117 (2014), arXiv:1209.4038 [hep-th].

[32] Nicolai Christiansen, Benjamin Knorr, Jan M. Pawlowski, and Andreas Rodigast, "Global Flows in Quantum Gravity," (2014), arXiv:1403.1232 [hep-th].

[33] Daniel Becker and Martin Reuter, "Propagating gravitons vs. 'dark matter' in asymptotically safe quantum gravity," JHEP 12, 025 (2014), arXiv:1407.5848 [hep-th].

[34] Daniel Becker and Martin Reuter, "En route to Background Independence: Broken split-symmetry, and how to restore it with bi-metric average actions," Annals Phys. 350, 225-301 (2014), arXiv:1404.4537 [hep-th].

[35] Daniel Becker and Martin Reuter, "Towards a $C$ function in 4D quantum gravity," JHEP 03, 065 (2015), arXiv:1412.0468 [hep-th].

[36] Christof Wetterich, "Exact evolution equation for the effective potential," Phys.Lett. B301, 90-94 (1993).

[37] Dario Benedetti, Kai Groh, Pedro F. Machado, and Frank Saueressig, "The Universal RG Machine," JHEP 1106, 079 (2011), arXiv:1012.3081 [hep-th].

[38] A. O. Barvinsky and G. A. Vilkovisky, "The Generalized Schwinger-Dewitt Technique in Gauge Theories and Quantum Gravity," Phys. Rept. 119, 1-74 (1985).

[39] Yves Decanini and Antoine Folacci, "Off-diagonal coefficients of the Dewitt-Schwinger and Hadamard representations of the Feynman propagator," Phys. Rev. D73, 044027 (2006), arXiv:gr-qc/0511115 [gr-qc].

[40] Damiano Anselmi and Anna Benini, "Improved Schwinger-DeWitt techniques for higher-derivative corrections to operator determinants," JHEP 10, 099 (2007), arXiv:0704.2840 [hep-th].

[41] Kai Groh, Stefan Rechenberger, Frank Saueressig, and Omar Zanusso, "Higher Derivative Gravity from the Universal Renormalization Group Machine," PoS EPS-
HEP2011, 124 (2011), arXiv:1111.1743 [hep-th].

[42] Kai Groh, Frank Saueressig, and Omar Zanusso, "Off-diagonal heat-kernel expansion and its application to fields with differential constraints," (2011), arXiv:1112.4856 [math-ph].

[43] "xAct: Efficient tensor computer algebra for Mathematica," http://xact.es/index.html, accessed: 2015-0730.

[44] J. M. Martín-García, R. Portugal, and L. R. U. Manssur, "The Invar tensor package," Computer Physics Communications 177, 640-648 (2007), arXiv:0704.1756 [cs.SC].

[45] J. M. Martín-García, D. Yllanes, and R. Portugal, "The Invar tensor package: Differential invariants of Riemann," Computer Physics Communications 179, 586590 (2008), arXiv:0802.1274 [cs.SC].

[46] J. M. Martín-García, "xPerm: fast index canonicalization for tensor computer algebra," Computer Physics Communications 179, 597-603 (2008), arXiv:0803.0862 [cs.SC].

[47] David Brizuela, Jose M. Martin-Garcia, and Guillermo A. Mena Marugan, "xPert: Computer algebra for metric perturbation theory," Gen. Rel. Grav. 41, 2415-2431 (2009), arXiv:0807.0824 [gr-qc].

[48] T. Nutma, "xTras: A field-theory inspired xAct package for mathematica," Computer Physics Communications 185, 1719-1738 (2014), arXiv:1308.3493 [cs.SC].

[49] Daniel F. Litim, "Optimized renormalization group flows," Phys.Rev. D64, 105007 (2001), arXiv:hepth/0103195 [hep-th].

[50] Andreas Nink, "Field Parametrization Dependence in Asymptotically Safe Quantum Gravity," Phys.Rev. D91, 044030 (2015), arXiv:1410.7816 [hep-th].

[51] Maximilian Demmel and Andreas Nink, "Connections and geodesics in the space of metrics," Phys. Rev. D92, 104013 (2015), arXiv:1506.03809 [gr-qc].

[52] Roberto Percacci and Gian Paolo Vacca, "Search of scaling solutions in scalar-tensor gravity," Eur. Phys. J. C75, 188 (2015), arXiv:1501.00888 [hep-th].

[53] Kevin Falls, "Critical scaling in quantum gravity from the renormalisation group," (2015), arXiv:1503.06233 [hepth].

[54] Kevin Falls, "On the renormalisation of Newton's constant," Phys. Rev. D92, 124057 (2015), arXiv:1501.05331 [hep-th].

[55] Holger Gies, Benjamin Knorr, and Stefan Lippoldt, "Generalized Parametrization Dependence in Quantum Gravity," Phys. Rev. D92, 084020 (2015), arXiv:1507.08859 [hep-th].

[56] Peter Labus, Roberto Percacci, and Gian Paolo Vacca, "Asymptotic safety in $O(N)$ scalar models coupled to gravity," Phys. Lett. B753, 274-281 (2016), arXiv:1505.05393 [hep-th].

[57] Wataru Souma, "Nontrivial ultraviolet fixed point in quantum gravity," Prog. Theor. Phys. 102, 181-195 (1999), arXiv:hep-th/9907027 [hep-th].

[58] M. Reuter and Frank Saueressig, "Renormalization group flow of quantum gravity in the Einstein-Hilbert truncation," Phys. Rev. D65, 065016 (2002), arXiv:hepth/0110054 [hep-th].

[59] Dario Benedetti and Francesco Caravelli, "The Local potential approximation in quantum gravity," JHEP 1206 , 017 (2012), arXiv:1204.3541 [hep-th].

[60] Maximilian Demmel, Frank Saueressig, and Omar Zanusso, "Fixed-Functionals of three-dimensional Quantum Einstein Gravity," JHEP 11, 131 (2012), 
arXiv:1208.2038 [hep-th].

[61] Juergen A. Dietz and Tim R. Morris, "Asymptotic safety in the $\mathrm{f}(\mathrm{R})$ approximation," JHEP 1301, 108 (2013), arXiv:1211.0955 [hep-th].

[62] Juergen A. Dietz and Tim R. Morris, "Redundant operators in the exact renormalisation group and in the $\mathrm{f}(\mathrm{R})$ approximation to asymptotic safety," JHEP 07, 064 (2013), arXiv:1306.1223 [hep-th].

[63] Dario Benedetti, "On the number of relevant operators in asymptotically safe gravity," Europhys. Lett. 102, 20007 (2013), arXiv:1301.4422 [hep-th].

[64] Maximilian Demmel, Frank Saueressig, and Omar Zanusso, "Fixed Functionals in Asymptotically Safe Gravity," in Proceedings, 13th Marcel Grossmann Meeting on Recent Developments in Theoretical and Experimental General Relativity, Astrophysics, and Relativistic Field Theories (MG13) (2015) pp. 2227-2229, arXiv:1302.1312 [hep-th].

[65] Maximilian Demmel, Frank Saueressig, and Omar Zanusso, "RG flows of Quantum Einstein Gravity on maximally symmetric spaces," JHEP 06, 026 (2014), arXiv:1401.5495 [hep-th].

[66] Maximilian Demmel, Frank Saueressig, and Omar Zanusso, "RG flows of Quantum Einstein Gravity in the linear-geometric approximation," Annals Phys. 359, 141165 (2015), arXiv:1412.7207 [hep-th].

[67] I. Hamzaan Bridle, Juergen A. Dietz, and Tim R. Morris, "The local potential approximation in the background field formalism," JHEP 1403, 093 (2014), arXiv:1312.2846 [hep-th].

[68] Juergen A. Dietz and Tim R. Morris, "Background independent exact renormalization group for conformally reduced gravity," JHEP 04, 118 (2015), arXiv:1502.07396 [hep-th].

[69] Julia Borchardt and Benjamin Knorr, "Global solutions of functional fixed point equations via pseudospectral methods," Phys. Rev. D91, 105011 (2015), arXiv:1502.07511 [hep-th].

[70] Nobuyoshi Ohta, Roberto Percacci, and Gian Paolo Vacca, "Flow equation for $f(R)$ gravity and some of its exact solutions," Phys. Rev. D92, 061501 (2015), arXiv:1507.00968 [hep-th].

[71] Nobuyoshi Ohta, Roberto Percacci, and Gian Paolo Vacca, "Renormalization Group Equation and scaling solutions for $\mathrm{f}(\mathrm{R})$ gravity in exponential parametrization," (2015), arXiv:1511.09393 [hep-th].

[72] Andreas Nink and Martin Reuter, "The unitary conformal field theory behind 2D Asymptotic Safety," (2015), arXiv:1512.06805 [hep-th]. 